\title{
Insight into the conformational stability of membrane-embedded BamA using a combined solution and solid-state NMR approach
}

\author{
Tessa Sinnige $\cdot$ Klaartje Houben · Iva Pritisanac $\cdot$ \\ Marie Renault $\cdot$ Rolf Boelens $\cdot$ Marc Baldus
}

Received: 8 October 2014/ Accepted: 17 December 2014/Published online: 8 January 2015

(C) Springer Science+Business Media Dordrecht 2014

\begin{abstract}
The $\beta$-barrel assembly machinery (BAM) is involved in folding and insertion of outer membrane proteins in Gram-negative bacteria, a process that is still poorly understood. With its 790 residues, BamA presents a challenge to current NMR methods. We utilized a "divide and conquer" approach in which we first obtained resonance assignments for BamA's periplasmic POTRA domains 4 and 5 by solution NMR. Comparison of these assignments to solid-state NMR (ssNMR) data obtained on two BamA constructs including the transmembrane domain and one or two soluble POTRA domains suggested that the fold of POTRA domain 5 critically depends on the interface with POTRA 4. Using specific labeling schemes we furthermore obtained ssNMR resonance assignments for residues in the extracellular loop 6 that is known to be crucial for BamAmediated substrate folding and insertion. Taken together,
\end{abstract}

Electronic supplementary material The online version of this article (doi:10.1007/s10858-014-9891-6) contains supplementary material, which is available to authorized users.

T. Sinnige $\cdot$ K. Houben $\cdot$ I. Pritisanac $\cdot$ M. Renault .

R. Boelens · M. Baldus $(\square)$

NMR Spectroscopy, Department of Chemistry, Faculty of

Science, Bijvoet Center for Biomolecular Research, Utrecht

University, Padualaan 8, $3584 \mathrm{CH}$ Utrecht, The Netherlands

e-mail: m.baldus@uu.nl

Present Address:

I. Pritisanac

Physical and Theoretical Chemistry Laboratory, South Parks

Road, Oxford OX1 3QZ, UK

Present Address:

M. Renault

Institute of Pharmacology and Structural Biology, 205 Route de

Narbonne, 31077 Toulouse Cedex 04, France our data provide novel insights into the conformational stability of membrane-embedded, non-crystalline BamA.

Keywords NMR spectroscopy - Membrane proteins . Proteoliposomes · $\beta$-Barrel assembly · Protein dynamics

\section{Introduction}

The outer membrane protein (OMP) BamA is the main component of the $\beta$-barrel assembly machinery (BAM) (Voulhoux et al. 2003; Wu et al. 2005) that folds and inserts OMPs into the outer membrane of Gram-negative bacteria, where they serve e.g. as porins, receptors and enzymes. In Escherichia coli, BamA consists of a transmembrane (TM) domain that is organized in a 16-stranded $\beta$-barrel, and a periplasmic extension containing five Polypeptide TRansport Associated (POTRA) domains (Noinaj et al. 2013; Sánchez-Pulido et al. 2003). The POTRA domains are thought to possess a chaperone function in binding the nascent OMP polypeptide (Kim et al. 2007; Knowles et al. 2008). Furthermore, recently solved crystal structures of BamA from Neisseria gonorrhoeae and Haemophilus ducreyi in bicelles (Noinaj et al. 2013) and two structures comprising the TM domain of E. coli BamA in detergent micelles (Albrecht et al. 2014; Ni et al. 2014) suggested that the $\beta$-barrel can open up to form a lateral gate for substrate release into the membrane. However, detailed investigations of dynamics and conformational changes will be required to reveal the molecular mechanism of BamA-mediated OMP folding and insertion.

In recent years, magic angle spinning (MAS) solid-state NMR (ssNMR) has proven a valuable method for structural studies of membrane proteins (see, e.g. Cady et al. 2010; Etzkorn et al. 2007, 2008, 2010; Lange et al. 2006; Shahid 
et al. 2012; van der Cruijsen et al. 2013; Wang et al. 2013) that can be probed in a native-like environment such as lipid bilayers, a feature that is not easily accessible by other structural biology techniques. The power of NMR spectroscopy is that atomic-resolution information on the structure, dynamics and interactions of the biomolecule of interest can be obtained. Previously, ssNMR studies from our lab showed that the periplasmic POTRA domains of BamA can undergo fast motion in a precipitated state or in membranes when POTRA 1-4 are removed (Renault et al. 2011). In contrast, our recent ssNMR experiments of membrane-embedded BamA suggested the absence of fast overall POTRA motion in constructs containing at least POTRA 4 and POTRA 5 (Sinnige et al. 2014a).

To study the structure and dynamics of membraneembedded BamA in more detail, extensive NMR resonance assignments are required. However, BamA encompasses 790 amino acids and hence represents a challenge for current ssNMR methods. Previously, we have demonstrated the use of combining solid-and solution-state NMR experiments to study the $40 \mathrm{kDa}$ histidine kinase DcuS (Etzkorn et al. 2008) and the dimeric $40 \mathrm{kDa}$ sensory rhodopsin/transducer complex (Etzkorn et al. 2010) in lipid bilayers. In the following, we have adapted such a "divide

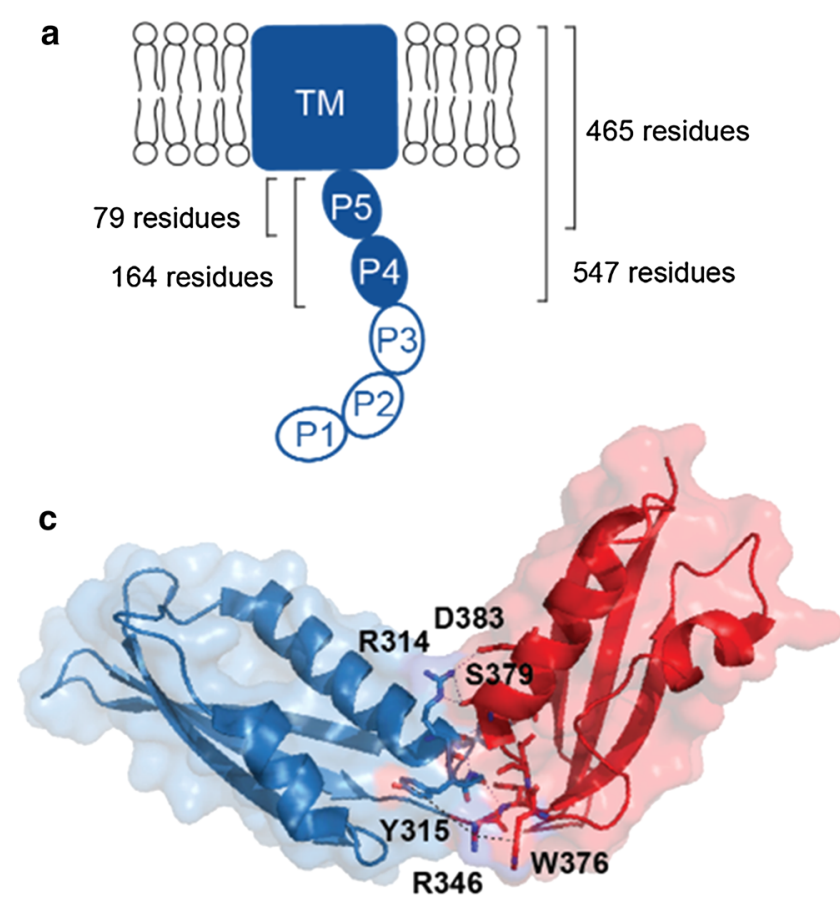

Fig. 1 a Constructs used in this study: isolated BamA P5 and P4P5 in solution, as well as membrane-embedded BamA P5-TM and BamA P4P5-TM, with their respective number of residues. TM transmembrane domain, $P$ POTRA. b $2 \mathrm{D}{ }^{1} \mathrm{H},{ }^{15} \mathrm{~N}$ HSQC spectra of BamA P5 (red) and P4P5 (blue) in solution (upper panel). Close-ups of the and conquer" approach (Fig. 1a) to study membraneembedded BamA. Firstly, we characterized the periplasmic POTRA domains 4 and 5 that precede the trans-membrane (TM) $\beta$-barrel domain using solution NMR. Assignments obtained in solution readily superimposed onto the ssNMR spectra of membrane-embedded BamA constructs. Using dedicated labeling schemes we subsequently succeeded in acquiring well-resolved 2D and 3D ssNMR spectra, leading to de-novo NMR assignments for a number of residues from the 390-residue BamA $\beta$-barrel. These studies shed light onto the conformational stability of BamA embedded in lipid bilayers, suggesting that in steady-state, the POTRA domains and extracellular loop 6 have a defined conformation, whereas the proposed lateral gate could not be detected in our experiments, possibly due to local motion or disorder.

\section{Materials and methods}

\section{Sample preparation}

The DNA fragments for BamA P5 (G344-N422) and P4P5 (T261-G424) were amplified from the plasmid pET11a $\Delta$ ssYaeT
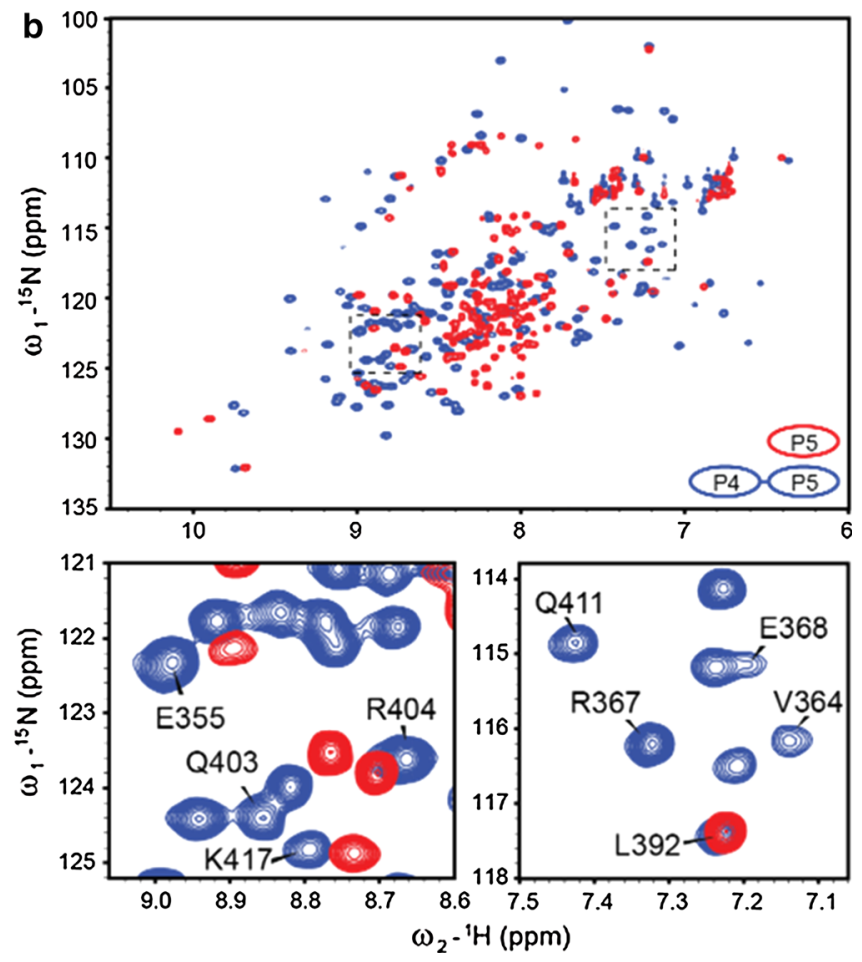

boxed regions (lower panels) show assignments for P5 residues in the P4P5 construct. c P4P5 crystal structure (PDB 3OG5) (GatzevaTopalova et al. 2010) with P4 in blue and P5 in red. Labeled residues contribute to the stabilizing interface by polar and cation- $\pi$ stacking interactions (dashed lines) 
encoding Escherichia coli bamA (Renault et al. 2011; Robert et al. 2006) and cloned into pLICHIS using enzyme-free cloning (de Jong et al. 2006) with the primers listed in Table 1. Proteins were produced in E. coli BL21 Rosetta in M9 minimal medium supplemented with $0.5 \mathrm{~g} / \mathrm{L}{ }^{15} \mathrm{NH}_{4} \mathrm{Cl}$ and in addition $2 \mathrm{~g} / \mathrm{L}{ }^{13} \mathrm{C}$ glucose for uniformly ${ }^{13} \mathrm{C},{ }^{15} \mathrm{~N}$-labeled samples. Cultures were grown at $37{ }^{\circ} \mathrm{C}$ and induced at the mid-exponential growth phase by adding IPTG at a final concentration of $1 \mathrm{mM}$, after which the culture was incubated at $25{ }^{\circ} \mathrm{C}$ for $16 \mathrm{~h}$. After cell lysis, the protein was purified in one step from the soluble fraction using nickel affinity chromatography. The His-tag was cleaved off after thrombin digestion and the sample was applied to a Superdex75 column (GE Healthcare) equilibrated in $50 \mathrm{mM}$ sodium phosphate pH 6.25 and $100 \mathrm{mM} \mathrm{NaCl}$, the buffer condition used in all solution NMR experiments. The proteins were concentrated to approximately $0.5 \mathrm{mM}$ for NMR measurements, using Amicon centrifugal concentrators (cut-off $3.5 \mathrm{kDa}$ ).

Construction of the plasmids pET11a $\Delta \mathrm{P} 1-\mathrm{P} 4 y a e T$ encoding BamA P5-TM and pET11c $\Delta \mathrm{P} 1-3 y a e T$ encoding BamA P4P5-TM was described previously (Renault et al. 2011; Sinnige et al. 2014a). Both proteins were produced in BL21(DE3) star as cytoplasmic inclusion bodies. Cells were grown at $37^{\circ} \mathrm{C}$ in minimal medium containing $100 \mu \mathrm{g} / \mathrm{mL}$ ampicillin. For reverse labeled cultures, the medium contained $2 \mathrm{~g} / \mathrm{L}{ }^{15} \mathrm{NH}_{4} \mathrm{Cl}$ and $2 \mathrm{~g} / \mathrm{L}{ }^{13} \mathrm{C}$-D-glucose and natural abundance amino acids were added $30 \mathrm{~min}$ prior to induction at a final concentration of $200 \mathrm{mg} / \mathrm{L}$. Forward labeled cultures contained the same concentration of natural abundance $\mathrm{NH}_{4} \mathrm{Cl}$ and D-glucose and $\mathrm{U}_{-}{ }^{13} \mathrm{C},{ }^{15} \mathrm{~N}$ labeled amino acids were added in the same way. Protein expression was induced by the addition of $1 \mathrm{mM}$ IPTG at an $\mathrm{OD}_{600}$ of $0.6-0.8$ and continued for $4 \mathrm{~h}$ at $37^{\circ} \mathrm{C}$.

Inclusion bodies were isolated as described previously (Dekker et al. 1995; Renault et al. 2011). BamA TM-P5 (VLK) was solubilized in $100 \mathrm{mM}$ glycine, $20 \mathrm{mM}$ Tris$\mathrm{HCl} \mathrm{pH} 8.0$ (IB buffer) containing $8 \mathrm{M}$ urea and refolded by 20 -fold dilution in $50 \mathrm{mM}$ Tris- $\mathrm{HCl}$ and $0.5 \% \mathrm{~N}$ dodecyl- $N, N$-dimethyl-3-ammonio-1-propanesulfonate (sulfobetaine 12, SB12), followed by overnight incubation at room temperature. All other BamA samples were solubilized in IB buffer containing $6 \mathrm{M}$ guanidinium chloride, refolded by tenfold dilution in $50 \mathrm{mM}$ sodium phosphate
pH 7.0 and $1 \% N, N$-dimethyl- $N$-dodecylamine- $N$-oxide (LDAO) and incubated overnight at room temperature.

For reconstitution, dilauroyl phosphatidylcholine (DLPC) (Avanti Polar Lipids) was dried from a chloroform solution to reach a molar lipid-to-protein ratio (LPR) of 21:1 for BamA P5-TM (VLK), 8:1 for BamA P5-TM (GSCAVLTI) and 10:1 for BamA P4P5-TM constructs, the latter two amounting to the same LPR in weight. The lipidic film was rehydrated with $20 \mathrm{mM}$ sodium phosphate and $5 \mathrm{mM} \mathrm{MgCl} 2$ (reconstitution buffer). Refolded protein was added and diluted approximately tenfold with reconstitution buffer, after which the mixed micelles were dialyzed in reconstitution buffer for 1 day at room temperature and several days at $4{ }^{\circ} \mathrm{C}$, until no detergent was observed in the dialysis buffer. The resulting proteoliposomes were collected by ultracentrifugation at $100,000 \times g$ for 1 or $2 \mathrm{~h}$ and the pellets were packed in $3.2 \mathrm{~mm}$ MAS rotors.

NMR spectroscopy

Solution NMR experiments were performed using AVANCE III Bruker spectrometers operating at $600 \mathrm{MHz}$ ${ }^{1} \mathrm{H}$ Larmor frequency equipped with regular or cryogenic TXI probes. Resonance assignments were obtained on $0.5 \mathrm{mM} \mathrm{U}-\left({ }^{13} \mathrm{C},{ }^{15} \mathrm{~N}\right)$-labeled $\mathrm{P} 4 \mathrm{P} 5$ in $50 \mathrm{mM}$ sodium phosphate $\mathrm{pH} 6.25,100 \mathrm{mM} \mathrm{NaCl}$ at $25{ }^{\circ} \mathrm{C}$ using standard $3 \mathrm{D}$ triple-(HNCA, HNcoCA, HNCACB, CBCAcoNH, HNCO, HAHBcoNH) and double-resonance (hCCH-DIPSY, HcCH-DIPSY) experiments. Secondary chemical shifts $\left(\mathrm{H}^{\mathrm{N}}, \mathrm{N}, \mathrm{HA}, \mathrm{CA}, \mathrm{CB}, \mathrm{C}^{\prime}\right)$ were computed using TALOS+ (Shen et al. 2009) and used for secondary structure determination. Resonance assignments were deposited in the BioMagResBank (BMRB) under accession code 19928.

All ssNMR experiments were performed on a Bruker AVANCE III spectrometer operating at $700 \mathrm{MHz}{ }^{1} \mathrm{H}$ Larmor frequency $(16.4 \mathrm{~T})$ and equipped with a $3.2 \mathrm{~mm}$ ${ }^{1} \mathrm{H},{ }^{13} \mathrm{C},{ }^{15} \mathrm{~N}$ MAS probe (Bruker BioSpin). The MAS frequency was set to $11 \mathrm{kHz}$ in experiments aimed at ${ }^{13} \mathrm{C},{ }^{13} \mathrm{C}$ sequential correlations (Seidel et al. 2004), and otherwise to $13 \mathrm{kHz}$ or $15 \mathrm{kHz}$. The effective sample temperature was kept to $-2{ }^{\circ} \mathrm{C}$ for dipolar-based experiments unless stated otherwise and to $8{ }^{\circ} \mathrm{C}$ for the INEPT-HETCOR experiment.
Table 1 Primers used to clone BamA P4P5 and P5 constructs

LIC overhangs are shown in capitals

\begin{tabular}{ll}
\hline BamA P4P5 T261 forward long & $5^{\prime}$ GCCGCGCGGCAGCCTGaccgaaggcgatcagtacaag 3' \\
BamA P4P5 T261 forward short & $5^{\prime}$ TGaccgaaggcgatcagtacaag 3' \\
BamA P4P5 G424 reverse long & $5^{\prime}$ CAAGAAGAACCCCTCAaccggtgttgcgctcttttacc 3' \\
BamA P4P5 G424 reverse short & $5^{\prime}$ TCAaccggtgttgcgctcttttacc 3' \\
BamA P5 G344 forward long & $5^{\prime}$ GCCGCGCGGCAGCCTGggtaaccgtttctacgtgcg 3' \\
BamA P5 G344 forward short & $5^{\prime}$ TGggtaaccgtttctacgtgcg 3' \\
BamA P5 N422 reverse long & $5^{\prime}$ CAAGAAGAACCCCTCAgttgcgctcttttaccttgtag 3' \\
BamA P5 N422 reverse short & $5^{\prime}$ TCAgttgcgctctttaccttgtag 3' \\
\hline
\end{tabular}


Fig. 2 a $\Delta \mathrm{C}_{\alpha}$ (upper panel) and $\Delta \mathrm{C}_{\beta}$ (lower panel) with $\Delta \mathrm{C}_{\alpha / \beta}$ $=\mathrm{C}_{\alpha / \beta}-\mathrm{C}_{\alpha / \beta \text { (random coil) }}$ for P4P5 residues plotted along the residue numbers. b Secondary structure of P4P5 from the crystal structure (PDB 3Q6B) and TALOS + predictions (Shen et al. 2009) based on solution NMR assignments
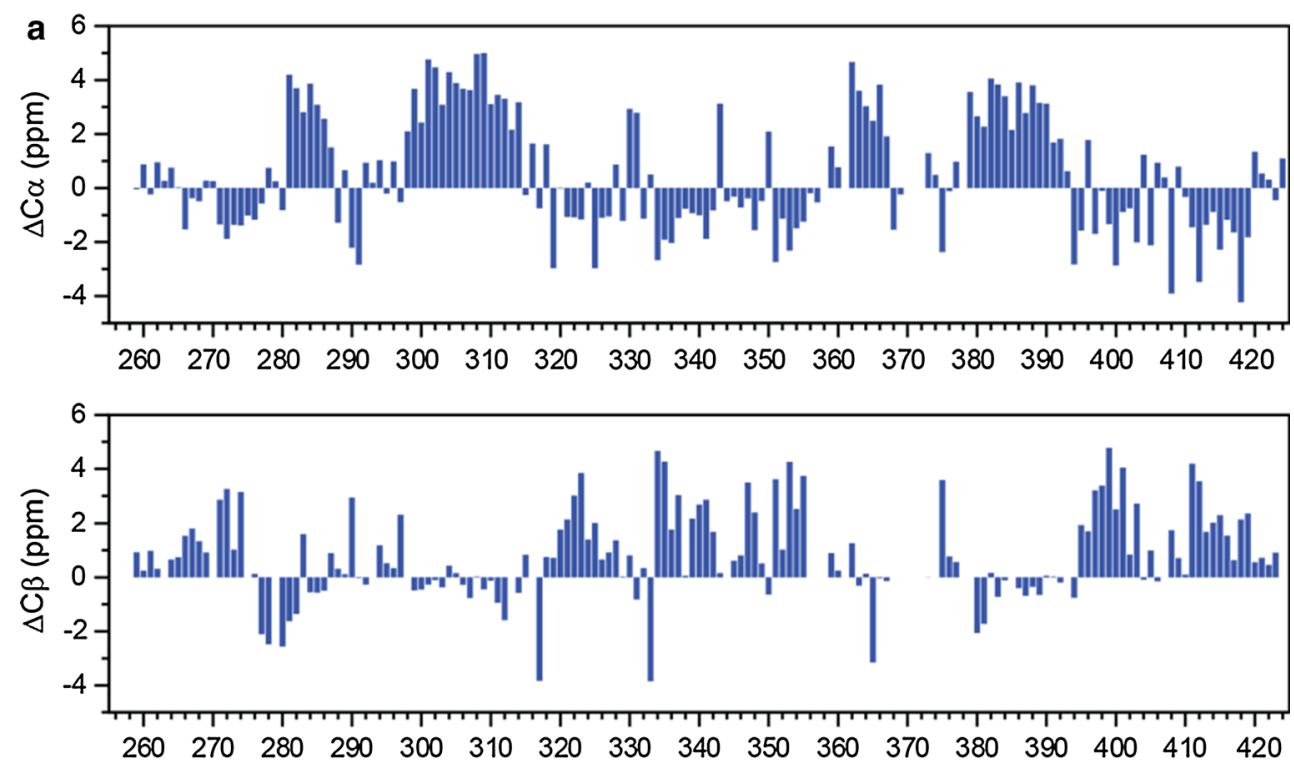

b

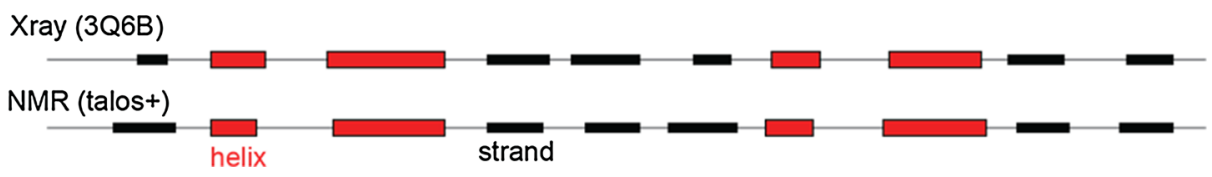

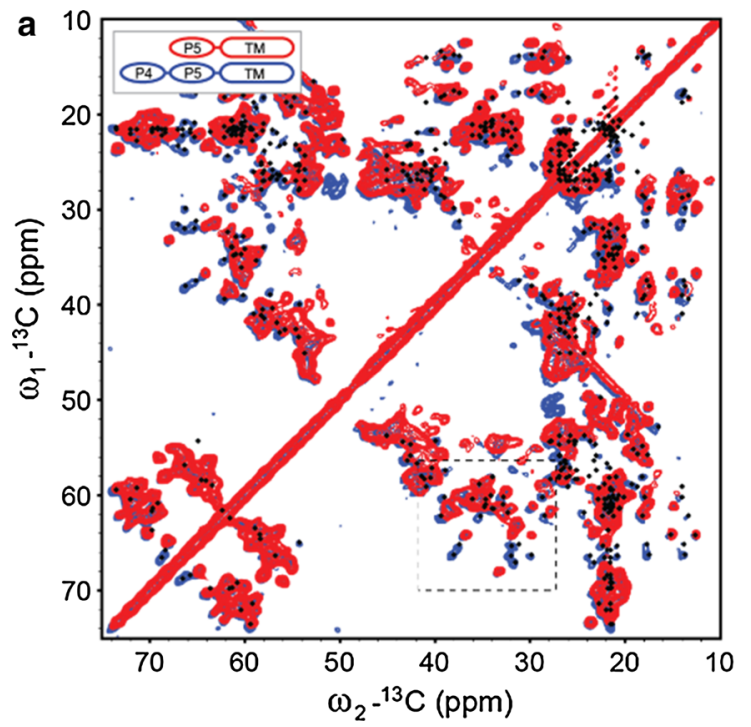
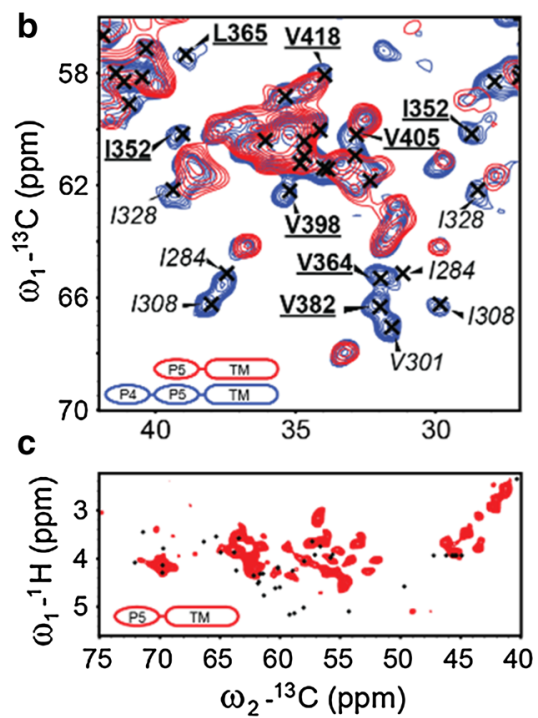

Fig. 3 a $2 \mathrm{D}{ }^{13} \mathrm{C},{ }^{13} \mathrm{C}$ PARIS ssNMR spectra of BamA P5-TM (red) and P4P5-TM (blue) (both ${ }^{13} \mathrm{C},{ }^{15} \mathrm{~N}$-labeled at amino acids GSAVLTI) in dilauroyl phosphatidylcholine (DLPC) bilayers. b Close-up of the boxed region in a. Black crosses are assignments for $\mathrm{P} 4 \mathrm{P} 5$ in solution. $\mathrm{P} 4$ peaks are labeled in italic, $\mathrm{P} 5$ bold and underlined. $\mathbf{c} \mathrm{C}_{\alpha}-\mathrm{H}_{\alpha}$ region

Hartman-Hahn cross-polarization (CP) was performed with a linear ramp of 70-100\%. The contact time was optimized for each sample and typically found between 600 and $900 \mu \mathrm{s} .{ }^{13} \mathrm{C},{ }^{13} \mathrm{C}$ magnetization transfer was achieved using the PARIS pulse sequence (Weingarth et al. 2009). $4 \mathrm{~ms}$ of a $2 \mathrm{D}{ }^{1} \mathrm{H},{ }^{13} \mathrm{C}$ INEPT-HETCOR ssNMR spectrum for mobile regions of BamA P5-TM $\left({ }^{13} \mathrm{C},{ }^{15} \mathrm{~N}\right.$-labeled at amino acids GSAVLTI $)$ in DLPC bilayers. Black dots indicate the expected correlations for P5 based on the solution NMR assignments

SPECIFIC-CP (Baldus et al. 1998) was used for ${ }^{15} \mathrm{~N}-{ }^{13} \mathrm{C}$ transfer steps. For decoupling, SPINAL64 (Fung et al. 2000) was applied with $78 \mathrm{kHz}$ irradiation on ${ }^{1} \mathrm{H}$. Spectra were processed using Bruker TopSpin 3.0 and analyzed in Sparky (Goddard and Kneller 2008). 


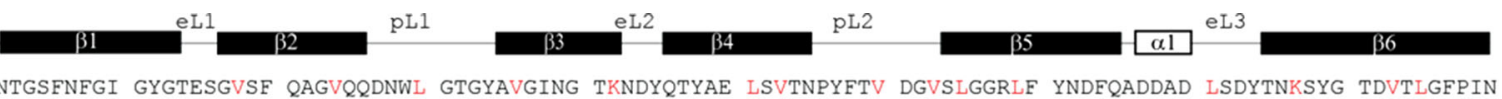

VLK forward RNTGSFNFGI GYGTESGVSF QAGVQQDNWL GTGYAVGING TKNDYQTYAE LSVTNPYFTV DGVSLGGRLF YNDFQADDAD LSDYTNKSYG TDVTLGFPIN GS (C) AVLTI RNTGSFNFGI GYGTESGVSF QAGVQQDNWL GTGYAVGING TKNDYQTYAE LSVTNPYFTV DGVSLGGRLF YNDFQADDAD LSDYTNKSYG TDVTLGFPIN

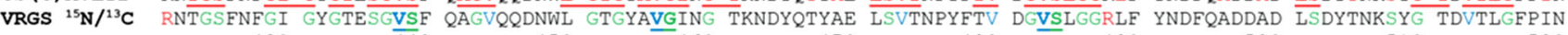
$\begin{array}{llllllll}430 & \overline{440} & 450 & 460 & 470 & 480 & 490 & 500\end{array}$

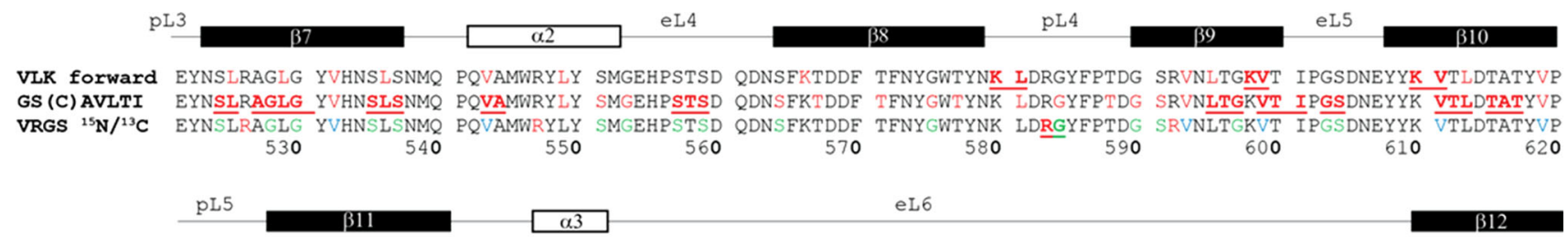

VLK forward IDDDHKWVVL GRTRWGYGDG LGGKEMPFYE NFYAGGSSTV RGFQSNTIGP KAVYFPHQAS NYDPDYDYEC ATQDGAKDLC KSDDAVGGNA MAVASLEFIT GS (C) AVLTI IDDDHKWVVL GRTRWGYGDG LGGKEMPFYE NFYAGGSSTV RGFQSNTIGP KAVYFPHQAS NYDPDYDYEC ATQDGAKDLC KSDDAVGGNA MAVASLEFIT VRGS ${ }^{15} \mathrm{~N} /{ }^{13} \mathrm{C}$ IDDDHKWVVL GRTRWGYGD LGGKEMPFYE NFYAGGSSTV RGFQSNTIGP KAVYFPHQAS NYDPDYDYEC ATQDGAKDLC KSDDAVGGNA MAVASLEFIT

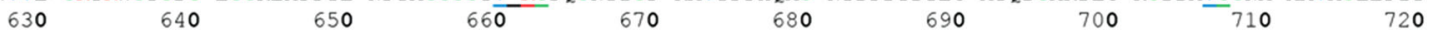

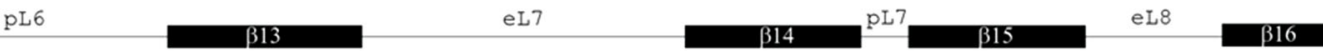

VLK forward PTPFISDKYA NSVRTSFFWD MGTVWDTNWD SSQYSGYPDY SDPSNIRMSA GIALQWMSPL GPLVESYAQP FKKYDGDKAE QFQFNIGKTW GS(C)AVLTI PTPFISDKYA NSVRTSFFWD MGTVWDTNWD SSQYSGYPDY SDPSNIRMSA GIALQWMSPL GPLVFSYAQP FKKYDGDKAE QFQFNIGKTW VRGS ${ }^{15} \mathrm{~N} /{ }^{13} \mathrm{C}$ PTPFISDKYA NSVRTSFFWD MGTVWDTNWD SSQYSGYPDY SDPSNIRMSA GIALQWMSPL GPLVFSYAQP FKKYDGDKAE QFQFNIGKTW

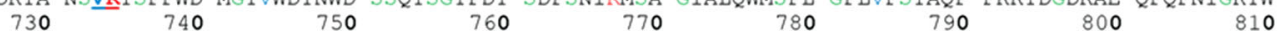

Fig. 4 ssNMR labeling schemes used in this study shown on the sequence of the BamA TM domain. The topology and secondary structural elements ( $\beta$-strands, $\alpha$-helices, periplasmic and extracellular loops, taken from crystal structure of the E. coli BamA TM domain, PDB 4N75) are indicated at the top. The residues are color

\section{Results}

The P5 domain of BamA occupies a central position in the BAM complex, being located at the interface of the BamA $\beta$-barrel and the membrane. Moreover, it serves as a binding site for the essential lipoprotein BamD and indirectly for the BamCDE sub-complex (Kim et al. 2007). To study this domain at atomic resolution, we firstly recorded NMR spectra of the isolated P5 domain and the tandem POTRA 4-5 (P4P5) construct in solution (Fig. 1a, b). Unexpectedly, the ${ }^{1} \mathrm{H},{ }^{15} \mathrm{~N}$ HSQC spectra, which serve as a fingerprint of the protein fold, showed large differences, indicating that the fold of P5 is not preserved between both constructs. Presumably, the correct fold of P5 requires stabilization by the P4P5 interface, consisting mainly of polar interactions and cation- $\pi$ interactions (Fig. 1c).

We obtained resonance assignments of P4P5 using standard solution NMR triple-resonance experiments (see "Materials and methods" section; BMRB deposition 19928). Backbone assignments could be obtained for 149 out of 159 non-proline residues. The $\mathrm{C}_{\alpha}$ and $\mathrm{C}_{\beta}$ secondary chemical shifts indicated the presence of secondary structure elements in line with previous crystallization studies using the P4P5 construct (Fig. 2) (Gatzeva-Topalova et al. 2010; Zhang et al. 2011). In contrast, the correlation pattern of the isolated P5 domain was reminiscent of a protein that is largely unfolded. Only the central $\beta$-sheet seemed to remain partially intact, judged by $\mathrm{P} 5$ resonances that remained close to the assignment in the P4P5 spectrum (Fig. 1b; Fig. S1a,b). coded according to the labeling: red ${ }^{13} \mathrm{C},{ }^{15} \mathrm{~N}$-labeled; blue ${ }^{13} \mathrm{C}$ labeled; green ${ }^{15} \mathrm{~N}$-labeled. Sequential contacts of labeled residues are shown underlined. Labeling schemes GS(C)AVLTI are combined into one row with cysteine residues in grey

Next, we examined whether the solution NMR assignments could serve as a reference for analysis of ssNMR spectra recorded on membrane-embedded BamA constructs. Moreover, we probed whether the fold of P5 would be stabilized by contacts with the periplasmic loops and the TM domain of BamA, which have been observed in crystal structures of BamA (Noinaj et al. 2013) and TamA (Gruss et al. 2013), another member of the Omp85 protein family. Hence, we investigated constructs consisting of the BamA TM domain preceded by either P5 alone or P4P5 in lipid bilayers using ssNMR. The protein variants were refolded from inclusion bodies and reconstituted in dilauroyl phosphatidylcholine (DLPC) bilayers at low lipid-to-protein ratio (LPR) to achieve the best possible sensitivity [see "Materials and methods" section and Sinnige et al. (2014a)].

We could readily identify signals of the $\mathrm{P} 4$ (italic) and P5 (bold and underlined) domains in ${ }^{13} \mathrm{C},{ }^{13} \mathrm{C}$ ssNMR correlation spectra of the P4P5-TM construct (Fig. 3a, b, blue) using our solution-state NMR assignments as a reference. In contrast, signals from P5 were not detected in dipolarbased ssNMR experiments on the P5-TM construct (Fig. 3a, b, red). In scalar-based experiments, which select for protein segments that are mobile on the nanosecond timescale (Andronesi et al. 2005; Renault et al. 2011), protein signals were observed but the peak positions did not correspond to the chemical shift assignments that we obtained in solution (Fig. 3c). Likely, this signal stems from unfolded or misfolded P5. Taken together, these findings confirmed that P5 folding critically depends on the 
Fig. 5 Assignments of unique sequential correlations in BamA P5-TM (VLK) reconstituted in DLPC at LPR 21:1 sample based on 2D ssNMR spectra. 2D ${ }^{13} \mathrm{C},{ }^{13} \mathrm{C}$ spectra were recorded with PARIS mixing times of $40 \mathrm{~ms}$ (blue, top) and $150 \mathrm{~ms}$ (black, top); the 2D NCACX (middle) and NCOCX (bottom) spectra were recorded with a $40 \mathrm{~ms}$ PARIS mixing time. Grey lines and bars indicate intra- and inter-residue correlations that led to the assignments that are labeled in the spectra. Experiments for intra-residue correlations $\left({ }^{13} \mathrm{C},{ }^{13} \mathrm{C}\right.$ with short mixing time and NCACX) were recorded at $15 \mathrm{kHz}$ MAS, whereas the experiments for sequential contacts were recorded at $11 \mathrm{kHz}$ MAS. In all experiments the effective sample temperature was kept at $-2{ }^{\circ} \mathrm{C}$

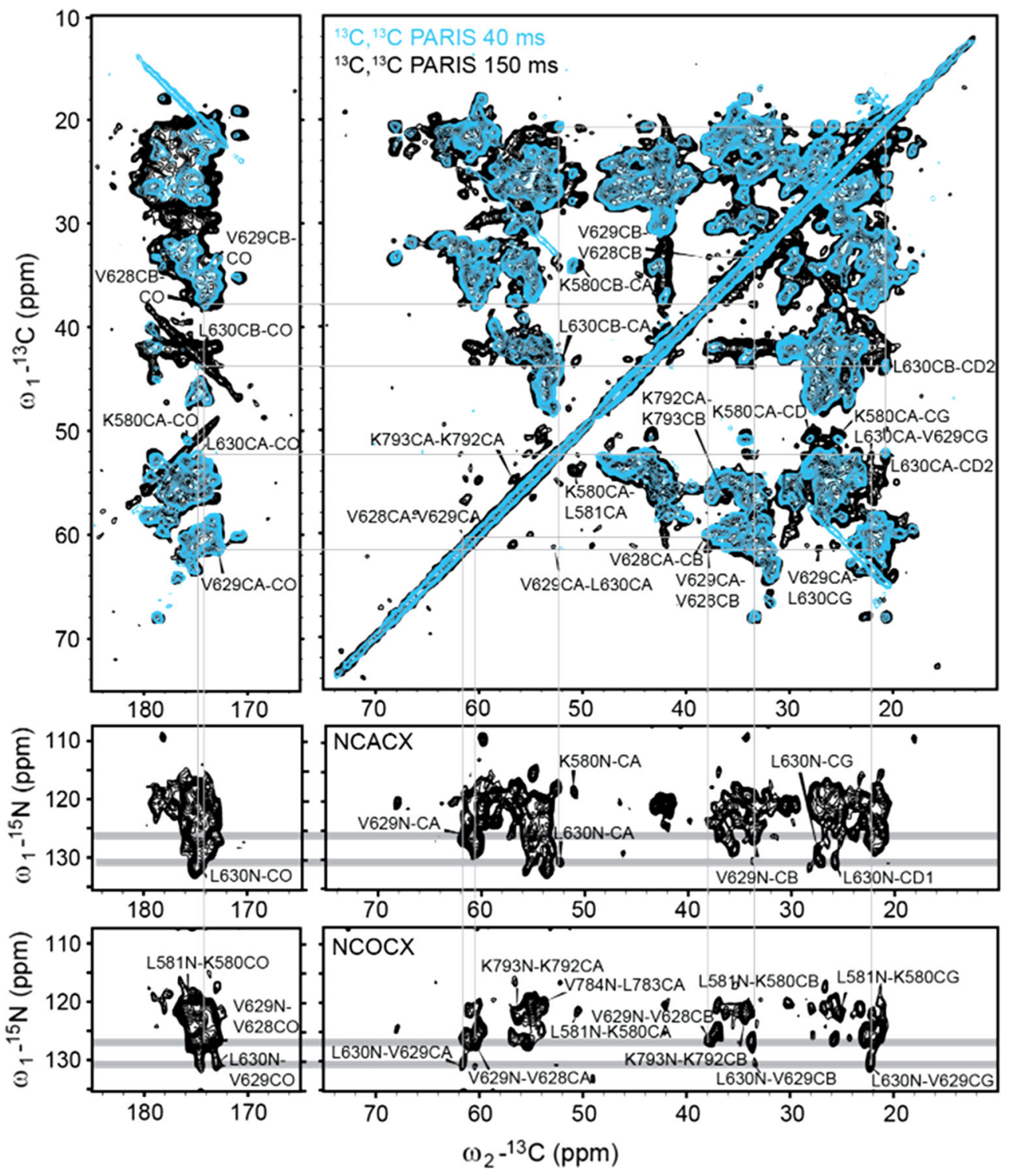

interface with $\mathrm{P} 4$, even when associated with the membrane-embedded BamA part and that the domains as a whole are relatively rigid in this sample preparation, as we observed in previous work (Sinnige et al. 2014a).

Finding unique sequential correlations using a minimal forward labeling scheme

We subsequently set out to obtain de-novo ssNMR resonance assignments of the membrane-embedded $\beta$-barrel domain of BamA. We initially chose to label only three residue types to obtain a minimum of sequential correlations (Fig. 4, first lane). For this forward labeling strategy, $\mathrm{U}_{-}{ }^{13} \mathrm{C},{ }^{15} \mathrm{~N}$ Val, Leu and Lys were added to the culture medium. The resulting BamA (VLK) sample should reveal the unique sequential contacts VVL, KK, LV and KL within the TM domain. To further limit spectral overlap in the dipolar-based spectra, we utilized the construct BamA P5-TM, in which signals from P5 were not observed as shown above.

Based on a set of 2D experiments, we could identify and partially assign the sequential correlations from residue pairs K580-L581, V628-V629-L630 and K792-K793 (Fig. 5). These residues are located in $\beta$-strand $8, \beta$-strand 11 , and the $\beta$-strand 15/extracellular loop 8 boundary region, respectively (vide infra).

More extended labeling scheme at high spectral resolution

Next, we extended the isotope labeling to other residue types. We selected residues GSCAVLTI because they can easily be identified by virtue of their ${ }^{13} \mathrm{C}$ chemical shift correlations (see Figs. 3, 4). We combined forward labeling of Thr and Ile and reverse labeling of the amino acids 

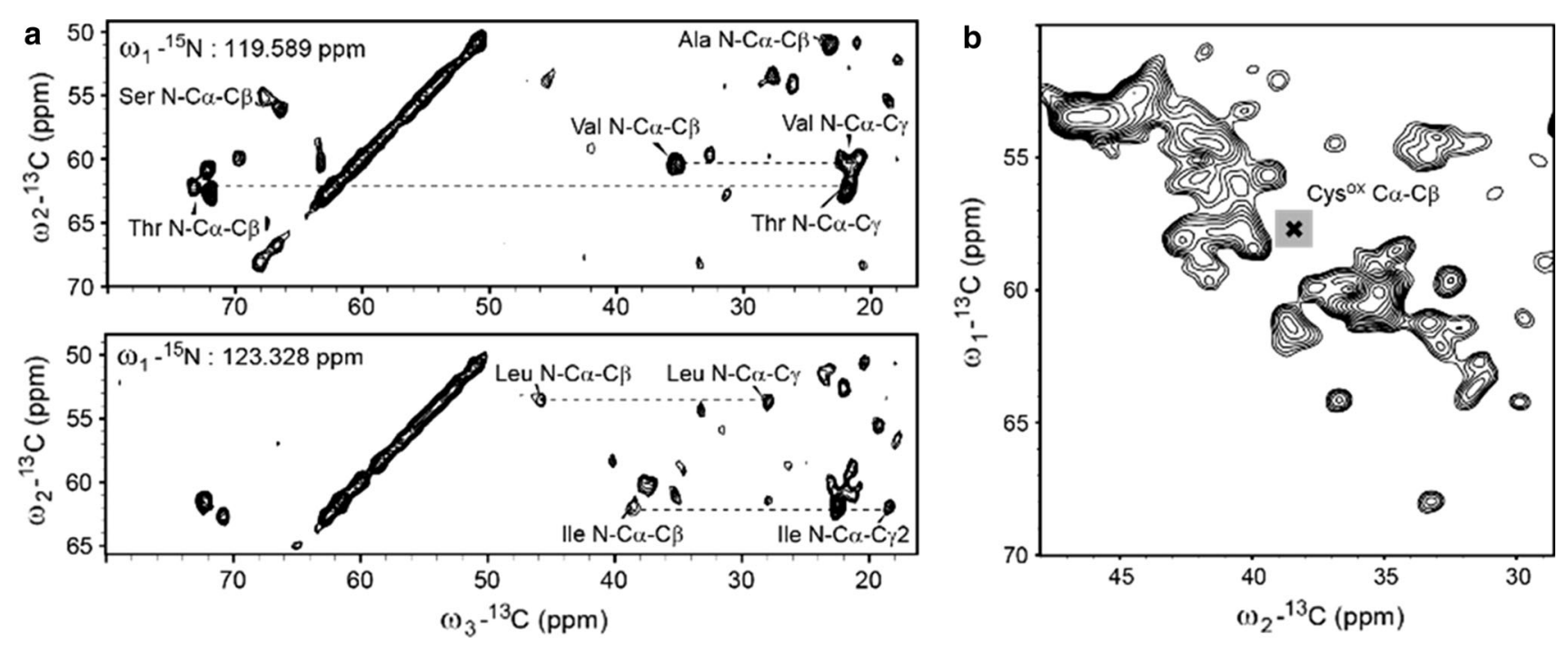

Fig. 6 a 2D planes extracted from the 3D NCACX spectrum recorded with a $30 \mathrm{~ms}$ PARIS mixing time on the BamA P5-TM (GSCAVLTI) sample reconstituted in DLPC (LPR of 8:1). b Close-up of a $2 \mathrm{D}{ }^{13} \mathrm{C},{ }^{13} \mathrm{C}$ experiment recorded with a $30 \mathrm{~ms}$ mixing time showing the expected position of the $\mathrm{C}_{\alpha}-\mathrm{C}_{\beta}$ correlation for oxidized

DEFHKMNPQRYW. Unfortunately, this strategy resulted in nitrogen scrambling. Indeed, glutamine and glutamate are subject to transamination in E. coli metabolism, and scrambling of the ${ }^{14} \mathrm{~N}$ atoms of these reverse labeled amino acids caused an overall decrease in ${ }^{15} \mathrm{~N}$ signal intensity. Consequently, the 3D NCOCX experiment was not feasible, but instead a 3D NCACX was recorded that was helpful to assign spin systems. This spectrum (Fig. 6a) was well resolved and individual spin systems could be traced to their respective amino-acid types. Interestingly, BamA contains only two cysteine residues located in the extracellular loop 6 (eL6) that presumably form a disulfide bond (Rigel et al. 2013). However, the chemical shift values that were measured experimentally did not correspond to oxidized cysteines (Fig. 6b), suggesting flexibility of this part of eL6 (vide infra).

Identification of residues in BamA's extracellular loop 6

Since the combination of reverse and forward labeling resulted in nitrogen scrambling, we subsequently opted for forward labeling of residues GSAVLTI. Because the two cysteine residues were not observed previously, this amino acid was not further included. This labeling strategy was applied to the BamA P4P5-TM construct. We note that including an additional POTRA domain did not affect the resonances from the TM domain (see Fig. 3a, b). cysteine in random coil conformation (black cross; grey box indicates the standard deviation) (Wang and Jardetzky 2002). The spectra were acquired at $13 \mathrm{kHz}$ MAS and an effective sample temperature of $2{ }^{\circ} \mathrm{C}$

By analyzing $2 \mathrm{D}{ }^{13} \mathrm{C},{ }^{13} \mathrm{C}$ spectra recorded with different mixing times (Fig. 7), the 3D NCACX discussed before (see Fig. 6), a 2D NCOCX data set (not shown), as well as a 3D NCOCX recorded on a sample with reverse labeling of the residues VLKY (not shown), the unique sequential stretches T667-I668-G669 and T600-I601 could be assigned. Remarkably, the stretch T667-I668-G669 is located in the extracellular loop 6 (eL6), which we speculated to be dynamic (see also Rigel et al. 2013). However, in the crystal structures of BamA from $N$. gonorrhoeae, $H$. ducreyi and E. coli, the loop partially folds back into the $\beta$-barrel (Albrecht et al. 2014; Ni et al. 2014; Noinaj et al. 2013). Notably, the assigned TIG stretch is very close to the conserved motif VRGF, which is locked to the wall of the $\beta$-barrel by several interactions in the crystal structures.

To examine eL6 and in particular the VRGF motif in more detail, we designed a tailored labeling strategy to be able to unambiguously assign these residues. We labeled BamA P4P5-TM with ${ }^{13} \mathrm{C}$-Val, ${ }^{13} \mathrm{C},{ }^{15} \mathrm{~N}$-Arg and ${ }^{15} \mathrm{~N}$-Gly and Ser, leading to $\mathrm{V}-\mathrm{R} / \mathrm{S} / \mathrm{G}$ and $\mathrm{R}-\mathrm{R} / \mathrm{G}$ sequential contacts in $2 \mathrm{D}$ NCO and NCOCX experiments (Fig. 8, see also Fig. 4). Comparison with solution NMR assignments of P4P5 corroborated the labeling strategy (Fig. 8, orange symbols). In addition, several cross-peaks were present that could be attributed to the TM domain, yet not all predictions matched well enough to serve as starting points for sequential assignment (Fig. 8, purple symbols). On the other hand, the 2D NCOCX experiment lacked many cross-peaks (Fig. 8, 


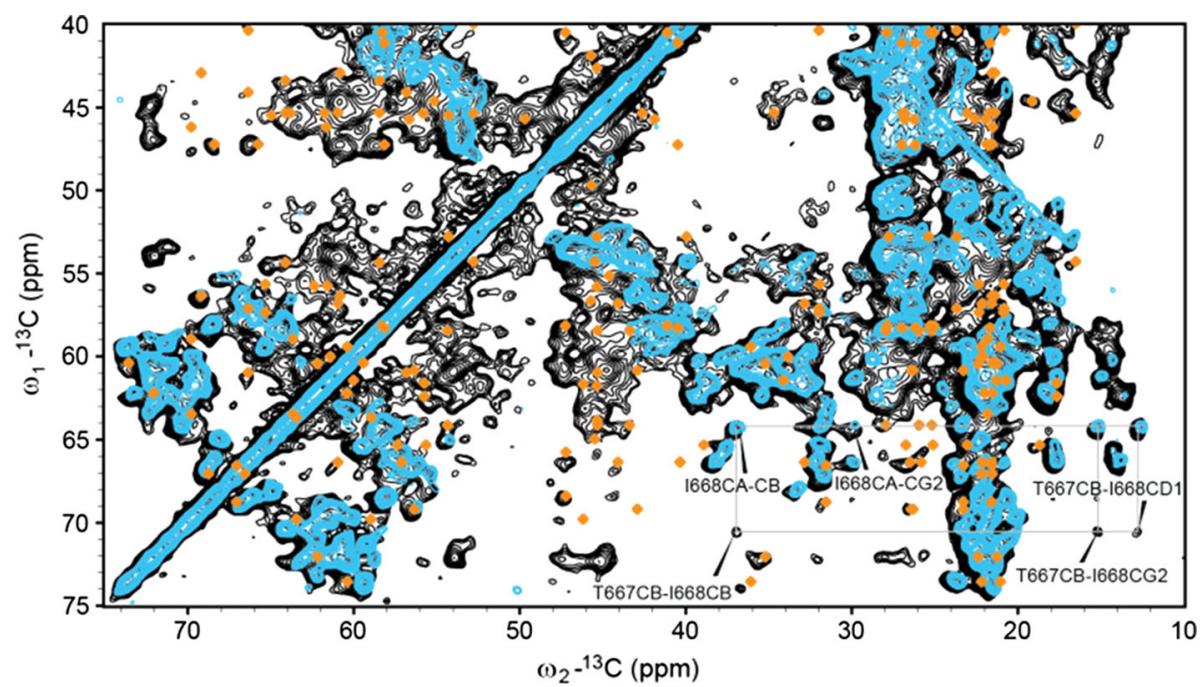

Fig. $72 \mathrm{D}{ }^{13} \mathrm{C},{ }^{13} \mathrm{C}$ correlation spectra recorded with $30 \mathrm{~ms}$ (cyan) and $150 \mathrm{~ms}$ (black) PARIS mixing times on the BamA P4P5-TM (GSAVLTI) sample reconstituted in DLPC (LPR 10:1). The sequential correlations between T667 and I668 are labeled and connected with grey lines. Orange dots indicate FANDAS predictions
(Gradmann et al. 2012) for sequential correlations based on solution NMR assignments of P4P5. The experiment with $30 \mathrm{~ms}$ mixing was recorded at $13 \mathrm{kHz}$ MAS frequency, whereas the experiment with $150 \mathrm{~ms}$ mixing was recorded at $11 \mathrm{kHz}$. In both cases the effective sample temperature was set to $-2{ }^{\circ} \mathrm{C}$

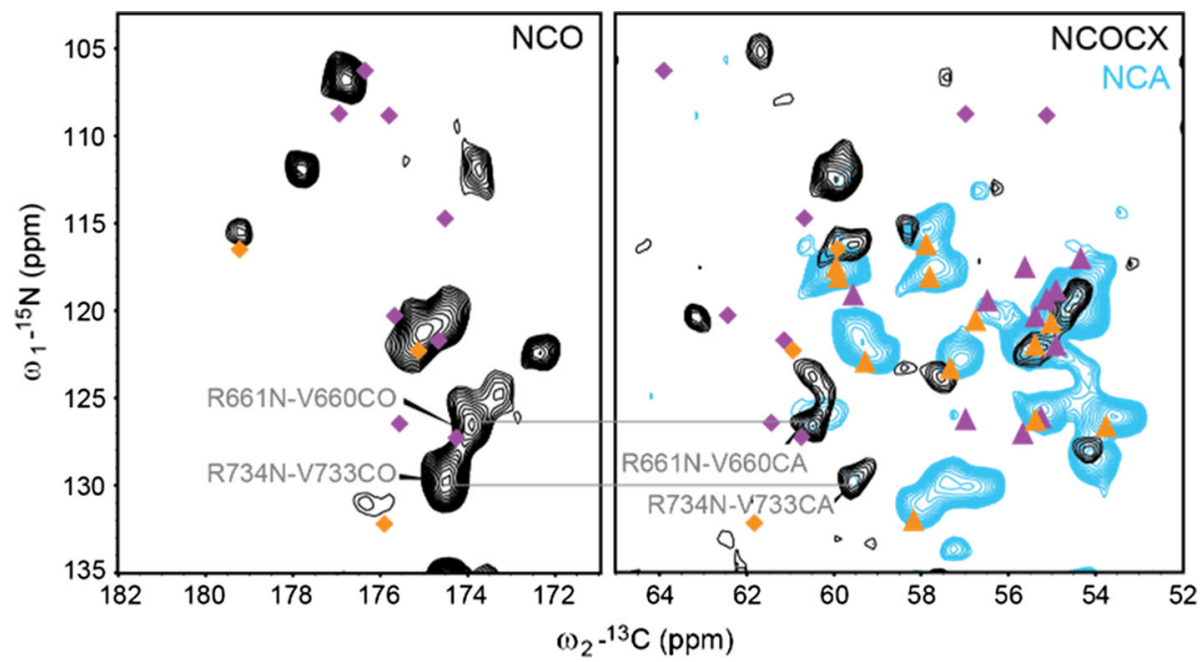

Fig. 8 Sequential correlations from BamA P4P5-TM labeled with ${ }^{13} \mathrm{C}-$ Val, ${ }^{13} \mathrm{C},{ }^{15} \mathrm{~N}$-Arg and ${ }^{15} \mathrm{~N}$-Gly and Ser and reconstituted in DLPC at LPR 10:1. Shown are a 2D NCO spectrum (left panel), and an overlay of a 2D NCOCX (right panel, black) and a 2D NCA (right panel, cyan). Squares and triangles represent predicted inter-residue and intra-residue correlations, respectively (orange: P4P5; purple: TM domain). Labels indicate tentative sequential assignments based on secondary structure. Spectra were recorded at $11 \mathrm{kHz}$ MAS (NCO, NCOCX) or $13 \mathrm{kHz}$ MAS (NCA) and an effective sample temperature of $4{ }^{\circ} \mathrm{C}$ right) due to the limited sensitivity of the experiment and potentially to the presence of protein dynamics. However, V660-R661 from the VRGF motif (random coil) and V733R734 ( $\beta$-strand) could be tentatively assigned based on their different secondary structures (Wang and Jardetzky 2002).

All ssNMR assignments obtained for the BamA TM domain from the different samples are listed in Table 2. The resulting conformation-dependent secondary chemical shifts based on $\mathrm{C}_{\alpha}$ and $\mathrm{C}_{\beta}$ assignments (Fig. 9a) are in good agreement with the crystal structure of the E. coli BamA TM domain (see Fig. 4) (Ni et al. 2014). The only exception is K793 that showed a $\beta$-strand rather than random coil value, but this residue is located at the tip of $\beta$-strand 15 (Fig. 9b). Possibly, this $\beta$-strand is slightly elongated in our proteoliposomal BamA preparations. In addition, the I668 residue located in eL6 showed a rather 
Table 2 ssNMR chemical-shift (CS) assignments of the BamA $\beta$ barrel in DLPC bilayers

\begin{tabular}{|c|c|c|c|c|c|}
\hline Residue & Atom & $\mathrm{CS}(\mathrm{ppm})$ & Residue & Atom & $\mathrm{CS}(\mathrm{ppm})$ \\
\hline K580 & $\mathrm{N}$ & 118.2 & L630 & $\mathrm{CA}$ & 52.3 \\
\hline K580 & CA & 50.9 & L630 & $\mathrm{CB}$ & 44.0 \\
\hline K580 & $\mathrm{CB}$ & 34.2 & L630 & CG & 27.2 \\
\hline K580 & $\mathrm{CD}$ & 28.0 & L630 & CD1 & 25.8 \\
\hline K580 & CG & 25.2 & L630 & CD2 & 20.6 \\
\hline K580 & CE & 43.2 & V660 & $\mathrm{CO}$ & 173.9 \\
\hline K580 & $\mathrm{CO}$ & 175.8 & V660 & $C A$ & 60.5 \\
\hline L581 & $\mathrm{N}$ & 121.3 & $R 661$ & $N$ & 126.6 \\
\hline L581 & CA & 54.0 & T667 & $\mathrm{CB}$ & 70.5 \\
\hline T600 & $\mathrm{CO}$ & 172.2 & I668 & $\mathrm{N}$ & 115.9 \\
\hline T600 & CA & 61.7 & I668 & CA & 64.2 \\
\hline T600 & $\mathrm{CB}$ & 72.3 & I668 & $\mathrm{CB}$ & 37.0 \\
\hline T600 & CG & 22.4 & I668 & CG1 & 30.0 \\
\hline I601 & $\mathrm{N}$ & 130.1 & I668 & CG2 & 15.2 \\
\hline I601 & CA & 61.3 & I668 & CD1 & 12.6 \\
\hline I601 & $\mathrm{CB}$ & 38.8 & I668 & $\mathrm{CO}$ & 176.6 \\
\hline I601 & CG1 & 29.9 & G669 & $\mathrm{N}$ & 111.8 \\
\hline I601 & CG2 & 17.7 & G669 & CA & 45.8 \\
\hline I601 & CD1 & 15.0 & $V 733$ & $C O$ & 174.5 \\
\hline V628 & CA & 60.2 & $V 733$ & $C A$ & 59.6 \\
\hline V628 & $\mathrm{CB}$ & 37.9 & $R 734$ & $N$ & 129.8 \\
\hline V629 & $\mathrm{N}$ & 126.7 & K792 & $\mathrm{CA}$ & 56.6 \\
\hline V629 & CA & 61.5 & K793 & $\mathrm{N}$ & 122.2 \\
\hline V629 & $\mathrm{CB}$ & 33.5 & K793 & CA & 54.8 \\
\hline V629 & CG & 22.2 & K793 & $\mathrm{CB}$ & 36.2 \\
\hline L630 & $\mathrm{N}$ & 130.5 & & & \\
\hline
\end{tabular}

Assignments with high confidence based on sequential correlations in at least two experiments; italics: tentative assignments based on secondary structure

$\alpha$-helical secondary chemical shift, although it has a random coil conformation in the crystal structures (Fig. 9b).

\section{Discussion}

Critical POTRA domain-domain interactions

in solution and solid-state

In this work, we applied a combination of solution and solidstate NMR experiments to obtain resonance assignments for the outer membrane protein BamA. We showed that solution NMR resonance assignments of the soluble construct comprising POTRA domains 4 and 5 superimpose well to the ssNMR data recorded on membrane-embedded BamA constructs. Remarkably, we found that P5 in isolation or attached to the $\beta$-barrel was not correctly folded, most likely due to the lack of a stabilizing interface with $\mathrm{P} 4$. It is interesting to note that BamA with only P5 is functional in $N$. meningitidis (Bos et al. 2007), suggesting that the requirement for stabilization by $\mathrm{P} 4$ can vary among different species. Contrary to our results, a recently published crystal structure of E. coli BamA P5-TM showed a folded P5 domain in the absence of P4 (Albrecht et al. 2014). In this structure however, P5 was highly stabilized by crystallographic contacts involving residues in exactly the region that we judged to be unfolded in our NMR experiments (Fig. 1b, Fig. S1b,c). This provides a likely explanation for the seemingly conflicting results and highlights the importance of studies on non-crystalline, membrane-embedded systems.

Solid-state NMR assignments of the BamA TM domain using specific labeling schemes

With its 390 residues, the TM domain of BamA is larger than any protein for which site-specific resonance assignments have been obtained using MAS ssNMR on unoriented samples. Moreover, we included one or two POTRA domains, leading to constructs of 465 and 547 residues, respectively (Fig. 1a). To limit spectral crowding and NMR assignment ambiguities, we used sparse labeling schemes with selectively labeled amino acids, VLK and GSAVLTI, that enabled the assignment of several short sequential stretches in the BamA TM domain based on 2D and 3D ssNMR experiments (Table 2). These residues are located in $\beta$ strands, at the beginning of extracellular loops and in the extracellular loop 6 (eL6) (Fig. 9b). Experimental secondary chemical shifts are in good agreement with secondary structural elements found in the crystal structure of the $E$. coli BamA TM domain (PDB 4N75), although $\beta$-strand 15 appeared elongated, as K793 was found to be in a $\beta$-strand conformation in our experiments, whereas it is the first residue of eL5 in the crystal structure (Fig. 9b).

More extended assignments could be achieved by labeling with precursors such as $1,3-{ }^{13} \mathrm{C}$ and $2-{ }^{13} \mathrm{C}$ glycerol (Higman et al. 2009) or $1-{ }^{13} \mathrm{C}$ and $2-{ }^{13} \mathrm{C}$ glucose (Loquet et al. 2011) that have been applied in ssNMR assignments of membrane proteins and fibrils. These methods have the advantage that spectral resolution is further improved by elimination of ${ }^{13} \mathrm{C}$ scalar couplings. Such labeling schemes can be expected to yield high-resolution spectra for BamA, but they rely on a well-characterized uniformly labeled sample and seem to be more useful in later stages of finalizing the assignments and collecting distance restraints for structure determination. Similar considerations may apply to the use of proton-detected solid-state NMR approaches that do not require membrane protein unfolding that we have already described in the context of BamA (Sinnige et al. 2014b) or the membrane-embedded ion channel KcsA (Weingarth et al. 2014). 

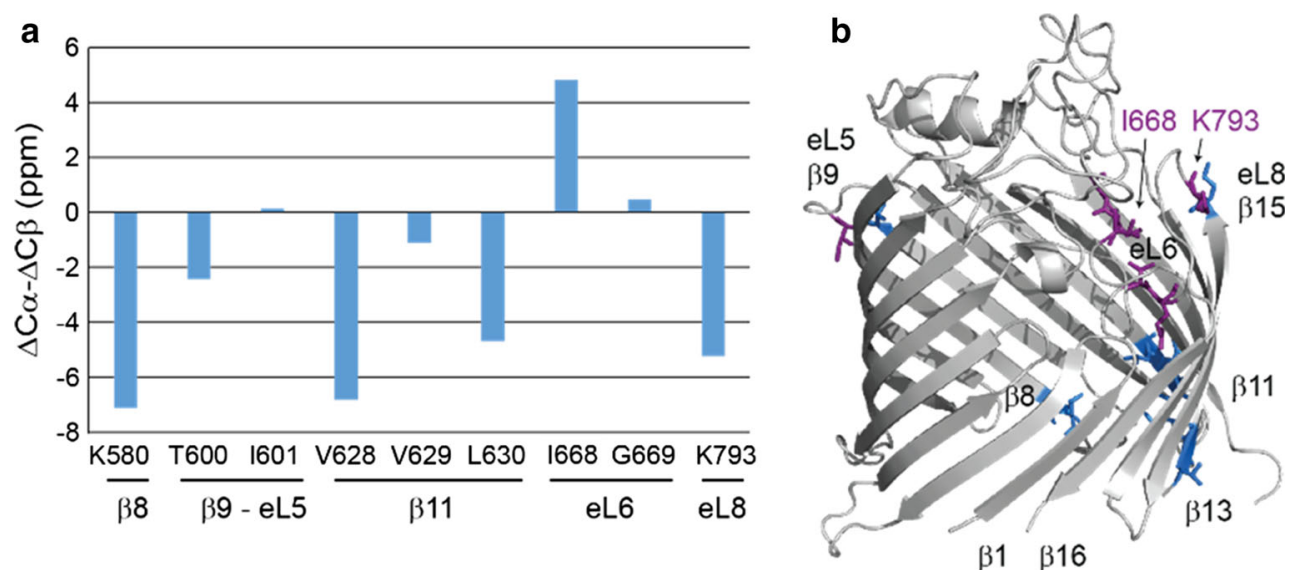

Fig. 9 a Secondary chemical shifts of BamA TM residues for which $\mathrm{C}_{\alpha}$ and $\mathrm{C}_{\beta}$ were assigned (or only $\mathrm{C}_{\alpha}$ for glycine). $\Delta \mathrm{C}_{\alpha / \beta}=\mathrm{C}_{\alpha / \beta}-$ $\mathrm{C}_{\alpha / \beta, \mathrm{rc}}$ (Wang and Jardetzky 2002). Positive values are indicative of $\alpha$-helical conformation; while negative ones suggest $\beta$-strand conformation. b Assigned residues are shown as sticks on the E. coli BamA TM domain (PDB 4N75) (Ni et al. 2014). Purple residues are located in extracellular loops $(\mathrm{eL})$; blue residues in $\beta$-strands
Fig. 10 a Overlay of available BamA crystal structures with eL6 colored (E. coli $\mathrm{PDB} 4 \mathrm{C} 4 \mathrm{~V}$ blue; E. coli $\mathrm{PDB} 4 \mathrm{~N} 75$ cyan; $N$. gonorrhoeae $\mathrm{PDB} 4 \mathrm{~K} 3 \mathrm{~B}$ purple; $H$. ducreyi $\mathrm{PDB} 4 \mathrm{~K} 3 \mathrm{C}$ yellow). The conserved VRGF/ $\mathrm{Y}$ motif is shown in sticks and the expected disulfide bond in E. coli BamA that is not resolved in the crystal structures is indicated with an orange line. b Labeling of $\beta$-strands 1 and 16 resulting from the amino acid schemes GSAVLTI (blue) and VLK (orange) shown in sticks on the E. coli BamA TM structure (PDB 4N75). Residues indicated in black form sequential correlations in one of the labeling schemes, whereas those in grey are isolated in the individual labeling schemes. c Hypothetical model of conformational changes within BamA. In steady-state, the BamA $\beta$-barrel is in equilibrium between an open and closed conformation, whereas eL6 (black) is folded back inside the $\beta$-barrel. In presence of a substrate (red), the POTRA domains may undergo a conformational change and eL6 is displaced

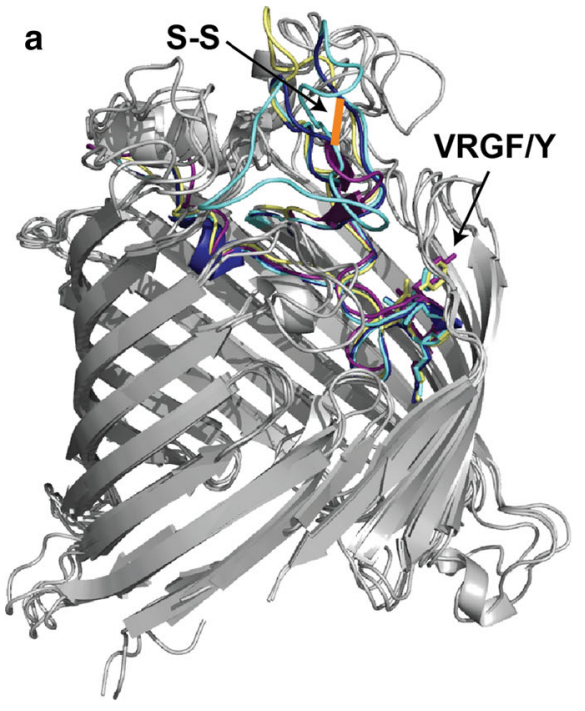

b
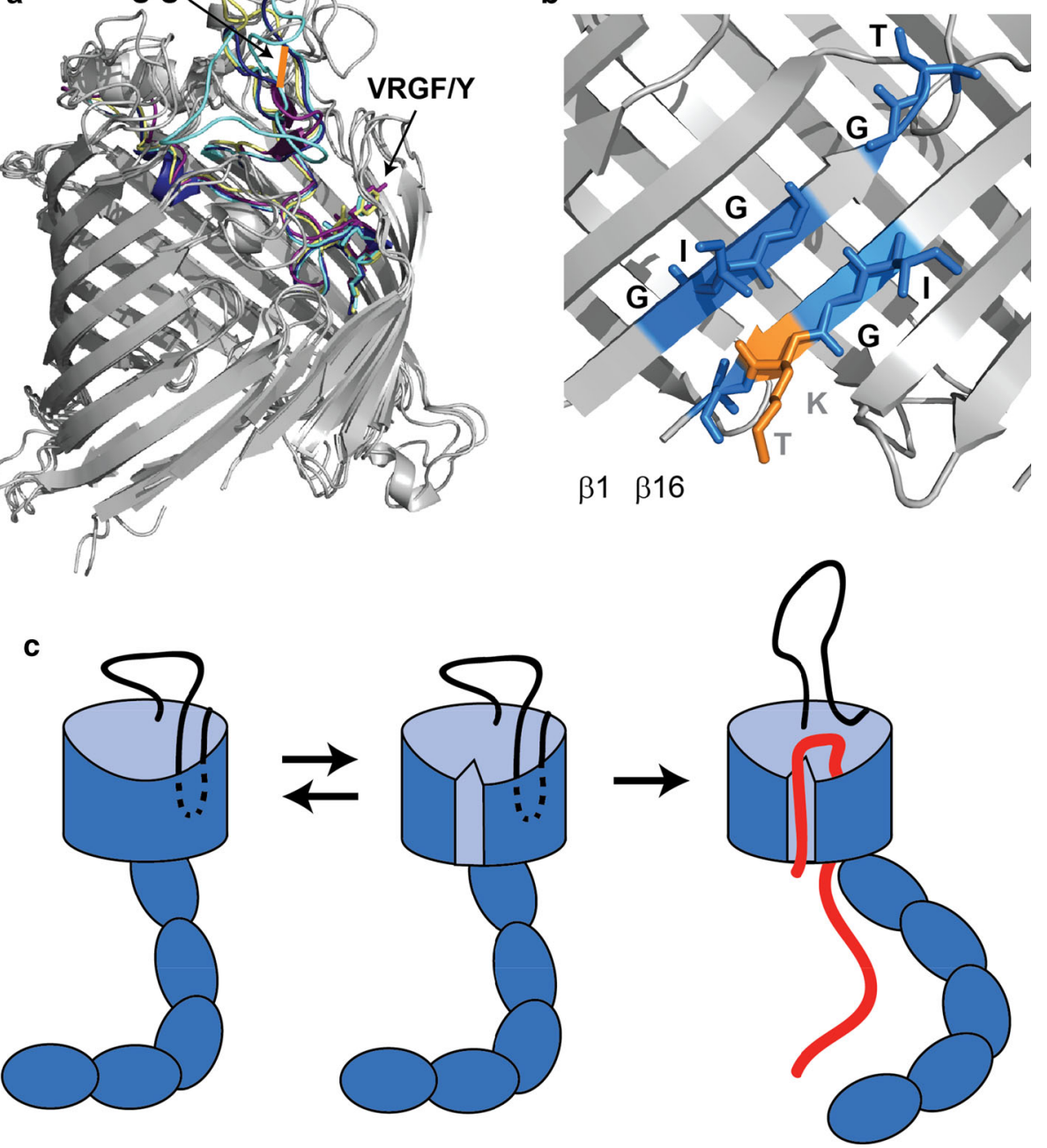
Implications for BamA dynamics

The long extracellular loop 6 is crucial for BamA function (Leonard-Rivera and Misra 2012; Noinaj et al. 2013) and has been proposed to undergo a conformational change related to substrate-insertion (Rigel et al. 2013). A short stretch from eL6, T667-I668-G669, was assigned using the GSAVLTI labeling strategy. We further examined this loop using an even sparser labeling scheme involving amino acids that are only ${ }^{15} \mathrm{~N}$ or ${ }^{13} \mathrm{C}$ labeled, and targeting at the conserved VRGF motif in eL6. Using this strategy, we obtained tentative assignments for V660-R661 from the conserved motif. These results suggest that this part of eL6 is rigid on a timescale of milliseconds and faster. In all BamA crystal structures currently available (Albrecht et al. 2014; Ni et al. 2014; Noinaj et al. 2013), eL6 similarly folds back into the $\beta$-barrel, where the conserved VRGF motif is stabilized by interactions with the $\beta$-barrel wall (Fig. 10a). Perhaps, binding of the substrate is required for a conformational change in eL6 to occur. Also the lipoproteins BamD and BamE have been implied in conformational cycling of BamA between a protected and exposed eL6 (Rigel et al. 2013). However, the part of eL6 that protrudes further away from the $\beta$-barrel may be more mobile in our sample preparations. The two cysteine residues that are present in the insertion in eL6 of E. coli BamA and supposedly form a disulfide bond (Rigel et al. 2013) were not observed in our experiments, in agreement with lacking electron density in the crystal structures (Fig. 10a).

Remarkably, most of the assigned residues map to the side of the BamA TM domain where eL6 is coordinated (Fig. 9b). Clearly, these results may be biased by the choice of labeling schemes and the fact that spectrally resolved chemical shifts can be more readily assigned, but they do imply that this part of the domain is altogether rigid. On the other hand, no residues around the proposed lateral gate (Noinaj et al. 2013) between $\beta$-strands 1 and 16 could be assigned in this study, although with the labeling schemes VLK and GSAVLTI, a significant portion of the terminal $\beta$-strands was labeled in our experiments (Fig. 10b). Whether these residues could not be assigned because of spectral crowding and sequence ambiguity, or as a consequence of dynamics (Fig. 10c), remains to be determined. A tailored labeling scheme to probe specific sequential residues in $\beta$-strands 1 and 16 , possibly in combination with proton detection (Sinnige et al. 2014a), should lead to further assignments.

Acknowledgments We thank Mark Daniels and Johan van der Zwan for excellent technical support. Scientific discussions with Dr. Markus Weingarth and Prof. Jan Tommassen are gratefully acknowledged. This work was funded by the Netherlands Organization for Scientific Research (NWO) (Grants 700.26.121 and 700.10.443 to M.B.).

\section{References}

Albrecht R et al (2014) Structure of BamA, an essential factor in outer membrane protein biogenesis. Acta Crystallogr Sect D 70:1779-1789. doi:10.1107/S1399004714007482

Andronesi OC, Becker S, Seidel K, Heise H, Young HS, Baldus M (2005) Determination of membrane protein structure and dynamics by magic-angle-spinning solid-state NMR spectroscopy. J Am Chem Soc 127:574-581

Baldus M, Petkova AT, Herzfeld J, Griffin RG (1998) Cross polarization in the tilted frame: assignment and spectral simplification in heteronuclear spin systems. Mol Phys 95:1197-1207. doi:10.1080/00268979809483251

Bos MP, Robert V, Tommassen J (2007) Functioning of outer membrane protein assembly factor Omp85 requires a single POTRA domain. EMBO Rep 8:1149-1154. doi:10.1038/sj. embor.7401092

Cady SD, Schmidt-Rohr K, Wang J, Soto CS, DeGrado WF, Hong M (2010) Structure of the amantadine binding site of influenza M2 proton channels in lipid bilayers. Nature 463:689-692

de Jong RN, Daniels MA, Kaptein R, Folkers GE (2006) Enzyme free cloning for high throughput gene cloning and expression. J Struct Funct Genomics 7:109-118. doi:10.1007/s10969-0069014-z

Dekker N, Merck K, Tommassen J, Verheij HM (1995) In vitro folding of Escherichia coli outer-membrane phospholipase A. Eur J Biochem 232:214-219

Etzkorn M, Martell S, Andronesi OC, Seidel K, Engelhard M, Baldus M (2007) Secondary structure, dynamics, and topology of a seven-helix receptor in native membranes, studied by solid-state NMR spectroscopy. Angew Chem Int Ed 46:459-462. doi:10. 1002/anie.200602139

Etzkorn M et al (2008) Plasticity of the PAS domain and a potential role for signal transduction in the histidine kinase DcuS. Nat Struct Mol Biol 15:1031-1039. doi:10.1038/nsmb.1493

Etzkorn M, Seidel K, Li L, Martell S, Geyer M, Engelhard M, Baldus M (2010) Complex formation and light activation in membraneembedded sensory rhodopsin II as seen by solid-state NMR spectroscopy. Structure 18:293-300. doi:10.1016/j.str.2010.01. 011

Fung BM, Khitrin AK, Ermolaev K (2000) An improved broadband decoupling sequence for liquid crystals and solids. J Magn Reson 142:97-101. doi:10.1006/jmre.1999.1896

Gatzeva-Topalova PZ, Warner LR, Pardi A, Sousa MC (2010) Structure and flexibility of the complete periplasmic domain of BamA: the protein insertion machine of the outer membrane. Structure 18:1492-1501. doi:10.1016/j.str.2010.08.012

Goddard TG, Kneller DG (2008) SPARKY 3. University of California, San Francisco

Gradmann S et al (2012) Rapid prediction of multi-dimensional NMR data sets. J Biomol NMR 54:377-387. doi:10.1007/s10858-0129681-y

Gruss F, Zähringer F, Jakob RP, Burmann BM, Hiller S, Maier T (2013) The structural basis of autotransporter translocation by TamA. Nat Struct Mol Biol 20:1318-1320

Higman VA et al (2009) Assigning large proteins in the solid state: a MAS NMR resonance assignment strategy using selectively and extensively ${ }^{13} \mathrm{C}$-labelled proteins. J Biomol NMR 44:245-260. doi: 10.1007/s10858-009-9338-7

Kim S, Malinverni JC, Sliz P, Silhavy TJ, Harrison SC, Kahne D (2007) Structure and function of an essential component of the outer membrane protein assembly machine. Science 317:961-964. doi: $10.1126 /$ science. 1143993

Knowles TJ et al (2008) Fold and function of polypeptide transportassociated domains responsible for delivering unfolded proteins 
to membranes. Mol Microbiol 68:1216-1227. doi:10.1111/j. 1365-2958.2008.06225.x

Lange A, Giller K, Hornig S, Martin-Eauclaire M-F, Pongs O, Becker S, Baldus M (2006) Toxin-induced conformational changes in a potassium channel revealed by solid-state NMR. Nature 440:959-962. doi:10.1038/nature04649

Leonard-Rivera M, Misra R (2012) Conserved residues of the putative L6 loop of Escherichia coli BamA play a critical role in the assembly of $\beta$-barrel outer membrane proteins, including BamA itself. J Bacteriol 194:4662-4668. doi:10.1128/JB.00825-12

Loquet A, Lv G, Giller K, Becker S, Lange A $(2011){ }^{13} \mathrm{C}$ spin dilution for simplified and complete solid-state NMR resonance assignment of insoluble biological assemblies. J Am Chem Soc 133:4722-4725. doi:10.1021/ja200066s

Ni D et al (2014) Structural and functional analysis of the $\beta$-barrel domain of BamA from Escherichia coli. FASEB J. doi:10.1096/ fj. $13-248450$

Noinaj N et al (2013) Structural insight into the biogenesis of $\beta$-barrel membrane proteins. Nature 501:385-390

Renault M, Bos MP, Tommassen J, Baldus M (2011) Solid-state NMR on a large multidomain integral membrane protein: the outer membrane protein assembly factor BamA. J Am Chem Soc 133:4175-4177. doi:10.1021/ja109469c

Rigel NW, Ricci DP, Silhavy TJ (2013) Conformation-specific labeling of BamA and suppressor analysis suggest a cyclic mechanism for $\beta$-barrel assembly in Escherichia coli. Proc Nat Acad Sci USA 110:5151-5156. doi:10.1073/pnas.1302662110

Robert V, Volokhina EB, Senf F, Bos MP, Gelder PV, Tommassen J (2006) Assembly factor Omp85 recognizes its outer membrane protein substrates by a species-specific C-terminal motif. PLoS Biol 4:1984-1995. doi:10.1371/journal.pbio.0030377

Sánchez-Pulido L, Devos D, Genevrois S, Vicente M, Valencia A (2003) POTRA: a conserved domain in the FtsQ family and a class of beta-barrel outer membrane proteins. Trends Biochem Sci 28:523-526. doi:10.1016/j.tibs.2003.08.003

Seidel K, Lange A, Becker S, Hughes CE, Heise H, Baldus M (2004) Protein solid-state NMR resonance assignments from ${ }^{13} \mathrm{C},{ }^{13} \mathrm{C}$ correlation spectroscopy. Phys Chem Chem Phys 6:5090-5093

Shahid SA, Bardiaux B, Franks WT, Krabben L, Habeck M, van Rossum B-J, Linke D (2012) Membrane-protein structure determination by solid-state NMR spectroscopy of microcrystals. Nat Methods 9:1212-1217. doi:10.1038/nmeth.2248
Shen Y, Delaglio F, Cornilescu G, Bax A (2009) TALOS + : a hybrid method for predicting protein backbone torsion angles from NMR chemical shifts. J Biomol NMR 44:213-223. doi:10.1007/ s10858-009-9333-Z

Sinnige T, Weingarth M, Renault M, Baker L, Tommassen J, Baldus M (2014a) Solid-state NMR studies of full-length BamA in lipid bilayers suggest limited overall POTRA mobility. J Mol Biol 426:2009-2021. doi:10.1016/j.jmb.2014.02.007

Sinnige T, Daniëls M, Baldus M, Weingarth M (2014b) Proton clouds to measure long-range contacts between nonexchangeable side chain protons in solid-state NMR. J Am Chem Soc 136:4452-4455. doi:10.1021/ja412870m

van der Cruijsen EAW et al (2013) Importance of lipid-pore loop interface for potassium channel structure and function. Proc Nat Acad Sci USA 110:13008-13013. doi:10.1073/pnas.1305563110

Voulhoux R, Bos MP, Geurtsen J, Mols M, Tommassen J (2003) Role of a highly conserved bacterial protein in outer membrane protein assembly. Science 299:262-265. doi:10.1126/science. 1078973

Wang Y, Jardetzky O (2002) Probability-based protein secondary structure identification using combined NMR chemical-shift data. Protein Sci 11:852-861. doi:10.1110/ps.3180102.Structure

Wang S et al (2013) Solid-state NMR spectroscopy structure determination of a lipid-embedded heptahelical membrane protein. Nat Methods 10:1007-1012

Weingarth M, Demco DE, Bodenhausen G, Tekely P (2009) Improved magnetization transfer in solid-state NMR with fast magic angle spinning. Chem Phys Lett 469:342-348. doi:10. 1016/j.cplett.2008.12.084

Weingarth M, van der Cruijsen EAW, Ostmeyer J, Lievestro S, Roux B, Baldus M (2014) Quantitative analysis of the water occupancy around the selectivity filter of a $\mathrm{K}+$ channel in different gating modes. J Am Chem Soc 136(5):2000-2007. doi:10.1021/ja411450y

Wu T, Malinverni J, Ruiz N, Kim S, Silhavy TJ, Kahne D (2005) Identification of a multicomponent complex required for outer membrane biogenesis in Escherichia coli. Cell 121:235-245. doi:10.1016/j.cell.2005.02.015

Zhang H, Gao Z-Q, Hou H-F, Xu J-H, Li L-F, Su X-D, Dong Y-H (2011) High-resolution structure of a new crystal form of BamA POTRA4-5 from Escherichia coli. Acta Crystallogr Sect F 67:734-738. doi:10.1107/S1744309111014254 